\title{
Nanoscale
}

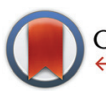

CrossMark \& click for updates

Cite this: Nanoscale, 2016, 8, 16694

Received 1st June 2016,

Accepted 29th August 2016

DOI: $10.1039 / c 6 n r 04430 a$

www.rsc.org/nanoscale

\section{Ordered gyroidal tantalum oxide photocatalysts: eliminating diffusion limitations and tuning surface barriers $\uparrow$}

\author{
Alexey S. Cherevan, ${ }^{a}$ Spencer Robbins, ${ }^{b}$ Dennis Dieterle, ${ }^{a}$ Paul Gebhardt, ${ }^{a}$ \\ Ulrich Wiesner ${ }^{\mathrm{b}}$ and Dominik Eder*a
}

\begin{abstract}
In this work we synthesized well-ordered, $\mathrm{Ta}_{2} \mathrm{O}_{5}$ films with a 3D-interconnected gyroid mesopore architecture with large pore sizes beyond $30 \mathrm{~nm}$ and extended crystalline domains through self-assembly of tailor-made triblock-terpolymers. This has effectively eliminated diffusion limitations inherent to previously reported mesoporous photocatalysts and resulted in superior hydrogen evolution with apparent quantum yields of up to $4.6 \%$ in the absence of any cocatalyst. We further show that the injection barrier at the solid-liquid interface constitutes a key criterion for photocatalytic performance and can be modified by the choice of the carbon template. This work highlights pore and surface engineering as a promising tool towards high-performance mesoporous catalysts and electrodes for various energyrelated applications
\end{abstract}

\section{Introduction}

Photocatalysis/photoelectrochemistry is central to a range of cutting-edge applications that address important socioeconomic areas such as energy (e.g. solar fuels, photovoltaics), environment (e.g. water and air purification, $\mathrm{CO}_{2}$ fixation) and health (e.g. disinfection, antibacterial coatings). Among the currently most sought-after applications is the photocatalytic oxidation and reduction of water ("artificial water splitting"), which is a process that converts energy of light photons into energy of chemical bonds ${ }^{1}$ and constitutes an important step for a potential hydrogen-based economy of the future. ${ }^{2-5}$ This requires a semiconductor with a suitable bandgap capable of absorbing light photons with sufficient energy to initiate the desired redox reactions. Another criterion is the lifetime of photoexcited charge carriers, which need to transfer to the catalyst's surface before they recombine. This can be addressed by improving crystallinity (i.e. diminishing grain boundaries and other structural defects, which can act as recombination centres) ${ }^{6}$ and through nanostructuring (i.e. shortening their

\footnotetext{
${ }^{a}$ Institut für Materialchemie, Technische Universität Wien, Getreidemarkt 9/BC/2, A-1060 Vienna, Austria. E-mail: dominik.eder@tuwien.ac.at

${ }^{b}$ Department of Materials Science and Engineering, 330 Bard Hall,

Cornell University, Ithaca, NY 14853, USA

$\dagger$ Electronic supplementary information (ESI) available: Supplementary figures (additional SEM and TEM images, long photocatalytic run), and the details of characterization methods and quantum yield calculations. See DOI: 10.1039/ c6nr04430a
}

diffusion paths to the solid-liquid interface). ${ }^{7}$ Most importantly, the photocatalyst needs to provide direct access to a large surface area without diffusion limitations.

Mesoporous materials engineering has emerged as a promising strategy for designing high-performance (photo)catalysts and (photo)electrodes in various energy related applications. ${ }^{8}$ Among the benefits of a mesoporous matrix are an increased active surface area and shorter diffusion paths for charge carriers. ${ }^{9}$ In addition, in contrast to random agglomerates of nanoparticles, materials with continuous porous networks are stable towards agglomeration in aqueous solutions. ${ }^{10}$

Various mesostructures have been produced by block copolymer self-assembly (e.g. with pluronics) ${ }^{11}$ ligand- or surfactant-assisted templating ${ }^{12}$ or by using anodized alumina as hard templates. ${ }^{13}$ Such structures have shown great promise in applications, such as dye-sensitized and perovskite solar cells,${ }^{15}$ in which efficient pore penetration and charge transport are required. So far, films have typically been reported with $0 \mathrm{D}$ cubic micellar and 1D hexagonally-ordered cylindrical architectures and were limited to comparatively small pore sizes (e.g. $5 \mathrm{~nm})$. The $1 \mathrm{D}$ access and small pore size generally induce kinetic limitations for reactant diffusion in liquid environments ${ }^{14}$ and as a consequence restrict the accessibility of the entire surface area for photocatalytic conversion.

In this work we aim to overcome these challenges by synthesizing well-ordered photocatalyst films with large, 3D-interconnected mesopores through self-assembly of tailormade triblock-terpolymers. We chose tantalum oxide $\left(\mathrm{Ta}_{2} \mathrm{O}_{5}\right)$ as a model compound, which is a highly active photocatalyst 
in the $U^{16}$ and - after conversion into (oxy)nitride - in the visible light range. ${ }^{17}$ So far, very few studies have been dedicated to mesoporous $\mathrm{Ta}_{2} \mathrm{O}_{5}$ albeit with limited success. ${ }^{18-22}$ In particular, we aim to design $3 \mathrm{D}$-gyroidal $\mathrm{Ta}_{2} \mathrm{O}_{5}$ structures with (a) pore sizes beyond $20 \mathrm{~nm}$ to facilitate reactant diffusion and enable direct access to a large inner surface without kinetic limitations, and (b) thin, highly-crystalline walls with large crystal domains to enhance charge transport to the solid-liquid interface and reduce charge recombination. We further envision that these structures will benefit other energy-related applications including (photo)electrochemical cells and batteries.

\section{Experimental section}

\section{Materials}

Hydrochloric acid (Aristar BDH, 37 wt $\%$, ACS/NF/FCC), tantalum(v) ethoxide (Sigma-Aldrich, 99.98\%, trace metals basis), tetrahydrofuran (Sigma-Aldrich, anhydrous, $\geq 99.9 \%$, inhibitor-free), chloroplatinic acid (Sigma-Aldrich, $8 \mathrm{wt} \%$ in water solution), toluene (Sigma-Aldrich, 99.8\%, anhydrous), ferrocene (Sigma-Aldrich, 98\%) and benzyl alcohol (SigmaAldrich, 99.8\%, anhydrous) were all used as received.

\section{Methods}

The block copolymer, poly(isoprene- $b$-styrene- $b$-ethylene oxide) (PI- $b$-PS- $b$-PEO; ISO), was synthesized by sequential anionic polymerization and characterized by a combination of gel permeation chromatography and nuclear magnetic resonance. ${ }^{23}$ In particular, the ISO used in this work had an overall molar mass of $69 \mathrm{~kg} \mathrm{~mol}{ }^{-1}$ and consisted of $29.6 \mathrm{wt} \%$ polyisoprene (I), $64.8 \mathrm{wt} \%$ polystyrene (S), and $5.6 \mathrm{wt} \%$ poly(ethylene oxide) (O) with a polydispersity index of 1.04 .

Gyroid $\mathrm{Ta}_{2} \mathrm{O}_{5}$ samples were prepared via the sol-gel process using hydrochloric acid as the catalyst. ISO was dissolved in tetrahydrofuran (THF) to yield a 2.7 wt $\%$ solution. The inorganic sol was prepared in a separate vial; $1 \mathrm{~mL}$ of THF $(24.66 \mathrm{mmol})$ was added to $0.343 \mathrm{~mL} \mathrm{HCl}$ and $1 \mathrm{~mL}$ (3.86 mmol) of $\mathrm{Ta}(\mathrm{OEt})_{5}$ was quickly injected while stirring vigorously. After $5 \mathrm{~min}, 1.28 \mathrm{~mL}$ of THF $(15.78 \mathrm{mmol})$ were quickly added to the solution, which was stirred for another 2 minutes. An aliquot of this sol was then added to the ISO solution to obtain a ratio of $0.34 \mathrm{~mL}$ sol per $100 \mathrm{mg}$ ISO. The solution was stirred again for 90 minutes and then poured into a PTFE dish to cast an ISO/Ta ${ }_{2} \mathrm{O}_{5}$ film. The PTFE dish was placed on a shallow glass crystallization dish, placed on a hotplate set to $50{ }^{\circ} \mathrm{C}$ and covered by a glass hemisphere to allow slow solvent evaporation and to initiate self-assembly. After complete solvent evaporation, the ISO/ $\mathrm{Ta}_{2} \mathrm{O}_{5}$ hybrid film was aged in a vacuum oven at $130{ }^{\circ} \mathrm{C}$ for several hours and subsequently calcined in ambient air at $700{ }^{\circ} \mathrm{C}$ for $2 \mathrm{~h}$ (ramp rate of $1 \mathrm{~K} \mathrm{~min}^{-1}$ ) to completely remove the polymer and induce crystallization. The reference sol-gel $\mathrm{Ta}_{2} \mathrm{O}_{5}\left(\mathrm{SG} \mathrm{Ta}_{2} \mathrm{O}_{5}\right)$ was synthesized following a similar procedure as described for the gyroid $\mathrm{Ta}_{2} \mathrm{O}_{5}$, however, without the addition of the polymer.
The procedure typically yielded around $30 \mathrm{mg}$ of the gyroid $\mathrm{Ta}_{2} \mathrm{O}_{5}$ (per batch), which was optimized to ensure uniform pore characteristics throughout the entire film $\left(\sim 1 \mathrm{~cm}^{2}\right.$, confirmed by statistics with SEM). In order to obtain comparatively large amounts of samples required for material characterization (e.g. $150 \mathrm{mg}$ for BET) and for photocatalytic tests $(50 \mathrm{mg})$, we prepared several batches and combined each of them after careful characterization with TEM/SEM, XRD and SAXS.

$\mathrm{Ta}_{2} \mathrm{O}_{5}$ nanotubes were prepared using CNTs as sacrificial templates. At first, MWCNTs were hybridized with $\mathrm{Ta}_{2} \mathrm{O}_{5}$ by means of a hydrothermally assisted sol-gel process to yield a $\mathrm{CNT}-\mathrm{Ta}_{2} \mathrm{O}_{5}$ hybrid with a thin layer of the oxide (see Fig. S2 $\dagger$ ). Importantly, benzyl alcohol was used to link the precursor molecules to the hydrophobic CNT surface. ${ }^{24}$ To obtain $\mathrm{Ta}_{2} \mathrm{O}_{5}$ NTs the nanocarbon was subsequently removed via heat treatment in air at $700{ }^{\circ} \mathrm{C}$ for $2 \mathrm{~h}\left(1 \mathrm{~K} \mathrm{~min}^{-1}\right)$ upon which the sample color turned white indicating oxidation of the CNTs.

\section{Photocatalytic experiments}

Water splitting experiments were performed using an inner irradiation gas flow slurry type home-build reactor consisting of two cylindrical parts, each double walled, the inner cylinder completely made of quartz (HSQ300 type from Heraeus) to be transparent to the UV portion of light. A medium pressure immersion type $700 \mathrm{~W} \mathrm{Hg}$ TQ 718 lamp (output power was adjusted to $500 \mathrm{~W}$ for all experiments) purchased from UV Consulting Company with an output range of 200-600 nm was inserted into the inner cylinder. In a single experiment $50 \mathrm{mg}$ of a powdered photocatalyst was dispersed in $10 \mathrm{vol} \% \mathrm{MeOH}-$ water solution by ultrasonication. The reaction medium was then transferred to the reactor. During the experiment, the reactor was continuously purged with argon 5.8 (flow rate of $100 \mathrm{ml} \mathrm{min}{ }^{-1}$, controlled with a mass flow controller Q-Flow 140 Series from MCC-Instruments). It was also used as a carrier gas for the online gas analyzer (X-Stream ${ }^{\circledR}$, Emerson Process Management). $\mathrm{H}_{2}$ was detected and quantified using the inbuilt thermal conductivity detector (TCD) that was calibrated with $\mathrm{H}_{2} / \mathrm{Ar}$ mixtures. The temperature of the reactor was kept at $10{ }^{\circ} \mathrm{C}$ through a water cooling system from Lauda (Variocool $1200 \mathrm{~W}$ ). Calculation of the apparent quantum yields (AQY) of the photocatalytic reaction is described in the ESI. $\dagger$

\section{Results and discussion}

Our strategy involved the preparation of ordered mesoporous $\mathrm{Ta}_{2} \mathrm{O}_{5}$ films with a $3 \mathrm{D}$ co-continuous gyroidal pore architecture (gyroid $\mathrm{Ta}_{2} \mathrm{O}_{5}$ ) using evaporation-induced self-assembly (EISA) of block copolymer directed sol-gel structures as schematically shown in Fig. 1a. Upon addition of an inorganic sol precursor, the hydrophilic block of the polymer swells, consequently altering the resulting polymer assembly relative to the parent polymer. The final pore structure can be controlled by synthesis parameters such as the water concentration, precursor/ copolymer ratio and evaporation kinetics. ${ }^{25}$ This process has 
a

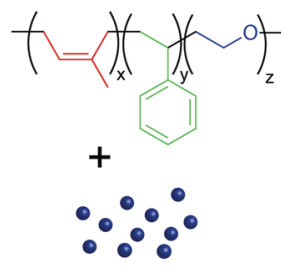

$\mathrm{ISO} / \mathrm{Ta}_{2} \mathrm{O}_{5}$ sol

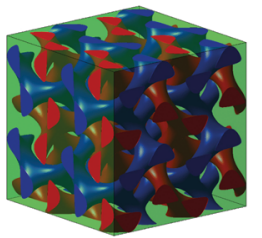

$\mathrm{ISO} / \mathrm{Ta}_{2} \mathrm{O}_{5}$ hybrid

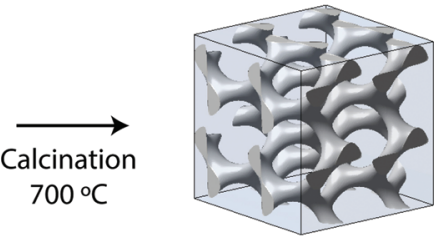

Mesoporous gyroid $\mathrm{Ta}_{2} \mathrm{O}_{5}$
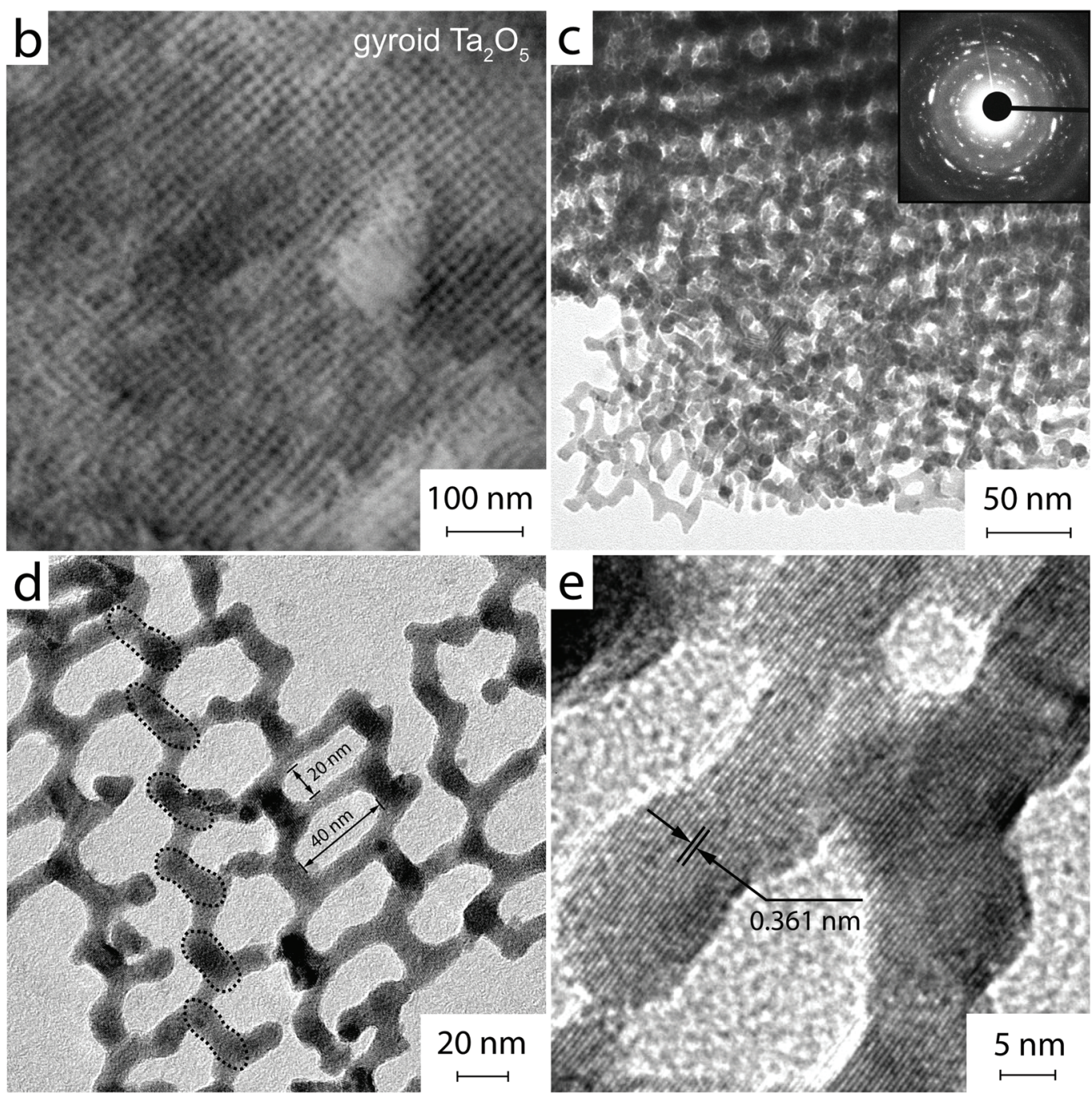

Fig. 1 (a) Illustration of the synthesis procedure of mesoporous $\mathrm{Ta}_{2} \mathrm{O}_{5}$ using ISO for self-assembly including casting the ISO/Ta $\mathrm{O}_{5}$ film from a precursor solution, slow solvent evaporation enabling ordered self-assembly, and calcination to remove the terpolymer and yield mesoporous $\mathrm{Ta}_{2} \mathrm{O}_{5}$. (b) SEM, (c) TEM, and (d), (e) HRTEM images of $\mathrm{Ta}_{2} \mathrm{O}_{5}$ samples with an ordered gyroid like structure with large pores and extended crystalline domains with monolithic character. The inset in (c) shows the ED pattern of the corresponding area. Dashed circles in (d) highlight the pore-to-pore distance (i.e. strut length) with uniform lengths of about $25 \mathrm{~nm}$. These areas share the same crystallographic orientation and are thus part of a larger single-crystalline domain.

been demonstrated for a wide range of metal oxides using commercial pluronic copolymers, e.g. P123 or F127. The synthesis of large, $>20 \mathrm{~nm}$, pores with 3D pore architectures, however, requires specifically designed terpolymers. In this work, we prepared a poly(isoprene)-b-poly(styrene)-b-poly(ethylene oxide) (PI- $b$-PS- $b$-PEO, ISO) terpolymer via anionic polymerization following Stefik et al. ${ }^{23}$ Volume fractions of the three blocks were optimized to yield a gyroid architecture upon addition of an inorganic sol according to the phase diagram of ISO. ${ }^{26,27}$

The ISO terpolymer was used to structure-direct the synthesis of mesoporous $\mathrm{Ta}_{2} \mathrm{O}_{5}$ films. As summarized in 
Fig. 1a, the terpolymer was first dissolved in THF and specific amounts of tantalum(v) ethoxide were added as a precursor for $\mathrm{Ta}_{2} \mathrm{O}_{5}$. After low-temperature solvent evaporation, the resulting films were calcined at $700{ }^{\circ} \mathrm{C}$ to remove the polymer scaffold (its oxidation occurs at about $400{ }^{\circ} \mathrm{C}$ ) and, consequently, convert the network into crystalline $\mathrm{Ta}_{2} \mathrm{O}_{5}$. We varied the composition of the sol as well as the polymer-to-inorganic ratio in order to achieve ordered mesopore structures.

Fig. $1 \mathrm{~b}$ shows a typical top-view SEM image of the $\mathrm{Ta}_{2} \mathrm{O}_{5}$ film, which reveals the presence of large, ordered pores. Both the pore diameter and pore arrangements are surprisingly uniform and representative of the macroscopic film (see Fig. S1 $\dagger$ ). TEM images in Fig. 1c and d suggest a 3D-interconnected network structure consistent with the alternating gyroid $\left(\mathrm{G}^{\mathrm{A}}\right)$ and pore sizes around $30 \mathrm{~nm}$. The walls are uniform in thickness of about $10 \mathrm{~nm}$, a preferred length scale for efficient light absorption and charge transport. Most importantly, the HRTEM image in Fig. 1e shows that the lattice fringes extend beyond the length of individual struts (indicated by the dashed circles in Fig. 1d), suggesting that the walls are composed of extended single-crystalline domains.
Small-angle X-ray scattering (SAXS) provides additional details on the mesostructure (Fig. 2a). The pattern of the ISO/ $\mathrm{Ta}_{2} \mathrm{O}_{5}$ hybrid exhibits reflections at $\left(d / d_{100}\right)^{2}=2,6,12,14$, and 22 , consistent with the alternating gyroid $\left(\mathrm{G}^{\mathrm{A}}\right)$ structure, and a $d_{100}$ spacing of $53.1 \mathrm{~nm} .{ }^{28}$ The SAXS data of the $700{ }^{\circ} \mathrm{C}$ treated gyroid $\mathrm{Ta}_{2} \mathrm{O}_{5}$ sample show significant changes in the ISO/ $\mathrm{Ta}_{2} \mathrm{O}_{5}$ hybrid. The primary reflection is shifted to a higher scattering vector, $q$, indicating a smaller $d_{100}$ of $29.9 \mathrm{~nm}$. This is associated with network shrinkage of about $40 \%$ as expected from comparable systems. ${ }^{14}$ Diminishing of higher order reflections and peak broadening suggest the loss of a longrange mesoscale order.

$\mathrm{X}$-ray diffraction (XRD, Fig. 2b) confirms that the network is crystalline without any noticeable amorphous content and consists of the $\beta-\mathrm{Ta}_{2} \mathrm{O}_{5}$ phase. ${ }^{29}$ The average crystal size, calculated by Scherrer's equation using the (100) diffraction peak, is about $26 \mathrm{~nm}$, which corresponds well to the average length of struts connecting network junctions of the $\mathrm{Ta}_{2} \mathrm{O}_{5}$ gyroid as observed by HRTEM (Fig. 1d). The dashed circles therefore indicate those sections within the extended crystalline domains that share the same crystallographic orientation and whose dimensions are guided by the wall thickness as well as
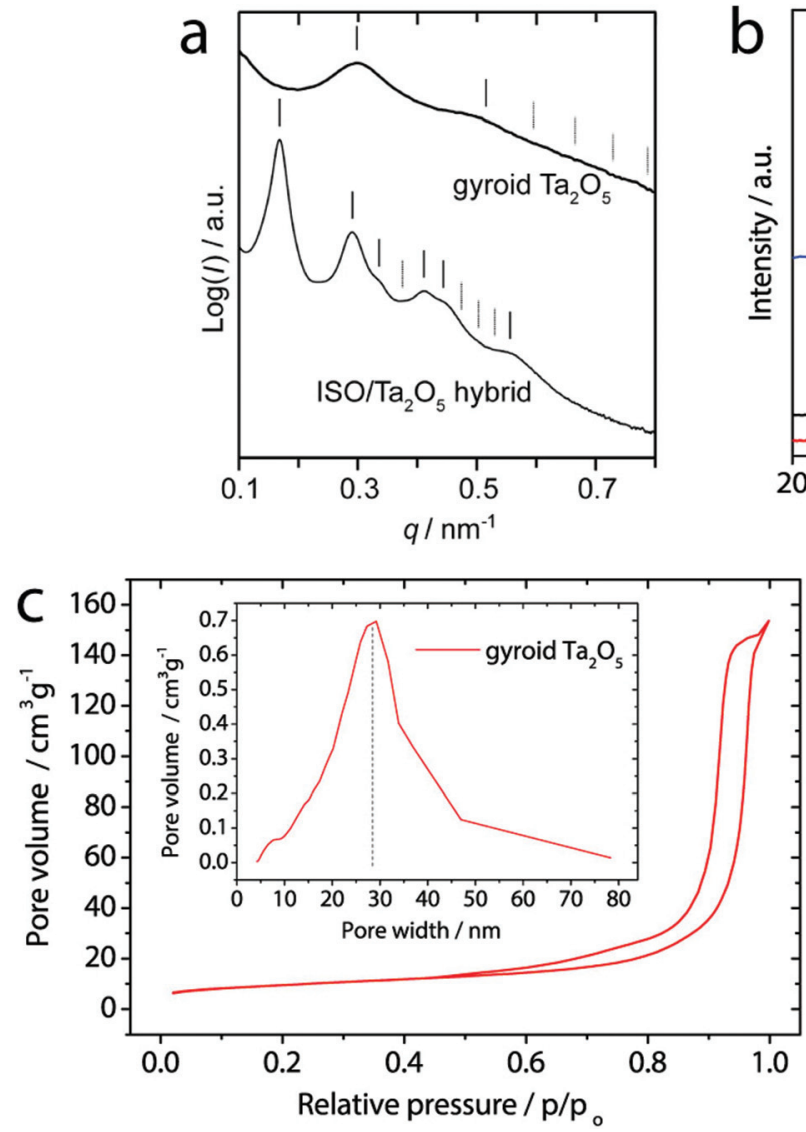
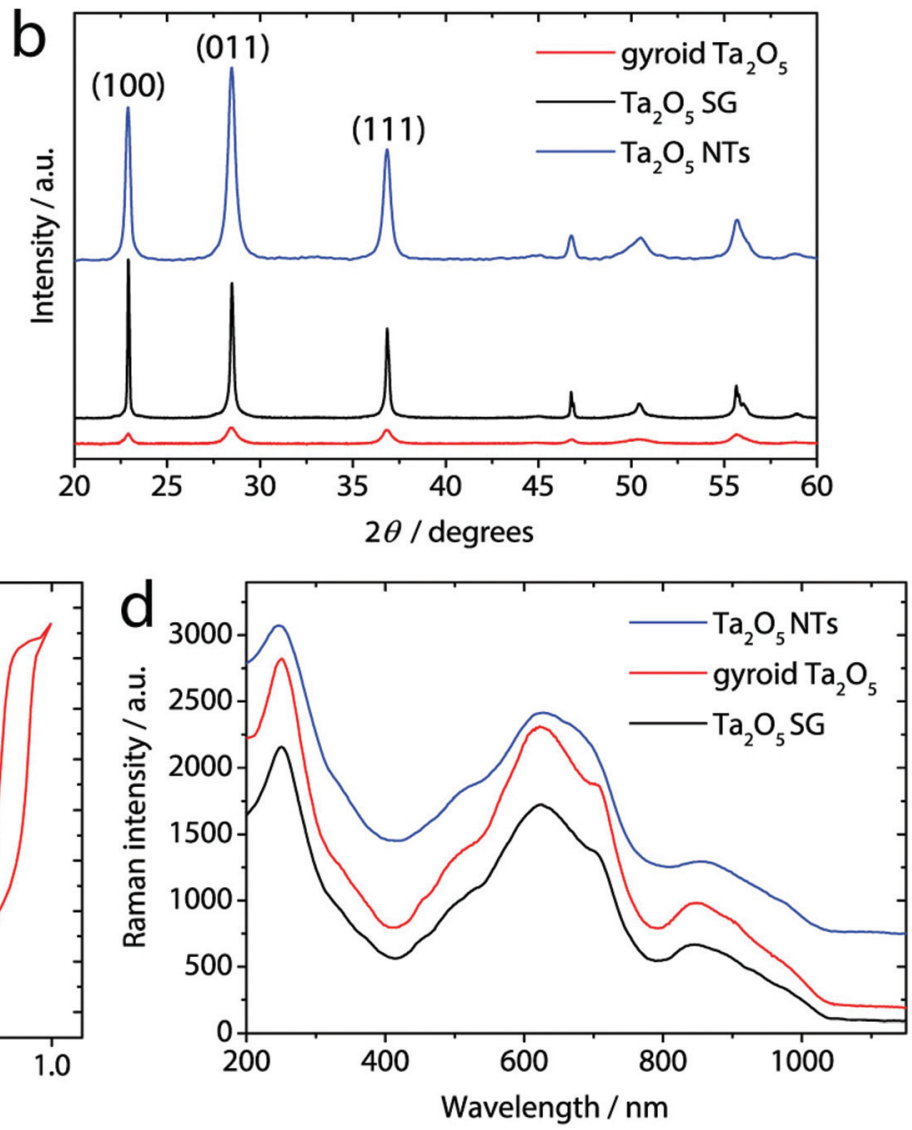

Fig. 2 (a) Azimuthally integrated SAXS patterns of the $\mathrm{ISO} / \mathrm{Ta}_{2} \mathrm{O}_{5}$ hybrid (bottom) and mesoporous gyroid $\mathrm{Ta}_{2} \mathrm{O}_{5}$ (top) samples. Solid vertical lines indicate the expected peak positions for the alternating gyroid $\left(G^{A}\right)$ morphology. (b) Comparison of the XRD patterns, and (d) Raman spectra (stacked) for the mesoporous gyroid $\mathrm{Ta}_{2} \mathrm{O}_{5}, \mathrm{Ta}_{2} \mathrm{O}_{5} \mathrm{NTs}$, and sol-gel $\mathrm{Ta}_{2} \mathrm{O}_{5}$ samples. (c) $\mathrm{N}_{2}$ sorption isotherms and the sorption isotherm derived pore size distribution of the mesoporous gyroid $\mathrm{Ta}_{2} \mathrm{O}_{5}$ sample. 
the pore-to-pore distance. $\mathrm{N}_{2}$ sorption studies reveal a type IV isotherm with $\mathrm{H} 2$ hysteresis (Fig. 2c), confirming the presence of mesopores. The sample exhibits a narrow BJH pore size distribution centred at $28 \mathrm{~nm}$. The specific surface area of $32 \mathrm{~m}^{2} \mathrm{~g}^{-1}$, according to BET, is considerably larger than that of a sol-gel derived reference sample $\left(\mathrm{Ta}_{2} \mathrm{O}_{5} \mathrm{SG}\right)$ produced without the terpolymer $\left(2.2 \mathrm{~m}^{2} \mathrm{~g}^{-1}\right)$.

The Raman spectra in Fig. $2 \mathrm{~d}$ and $\mathrm{S} 4 \dagger$ show the typical peaks of $\beta-\mathrm{Ta}_{2} \mathrm{O}_{5}$ without any secondary or impurity phases (e.g. tantalum carbides, carbon layer) as well as the absence of any peak shifts and/or broadening that are typically associated with the presence of defects. ${ }^{32}$

These results confirm the successful synthesis of crystalline $\mathrm{Ta}_{2} \mathrm{O}_{5}$ networks with alternating gyroidal pore structures, uniformly large pore sizes, and single-crystalline domains that extend beyond the strut limitations of the gyroidal network. Moreover, the combined results reveal no carbon residues within the detection limits.

The photocatalytic performance of the samples was evaluated for hydrogen evolution via sacrificial water splitting conducted in an inner irradiation type flow reactor, each using $50 \mathrm{mg}$ of the photocatalyst (see Experimental section for details). For comparison, a conventional non-porous $\mathrm{Ta}_{2} \mathrm{O}_{5} \mathrm{SG}$ powder was used as a reference (see Fig. $\mathrm{S} 1 \dagger$ and Experimental section). Fig. 3a reveals the remarkable photocatalytic activity of gyroid $\mathrm{Ta}_{2} \mathrm{O}_{5}$ samples towards water reduction without the use of any co-catalyst (e.g. peak and stabilized hydrogen evolution rates of 1188 and $955 \mu \mathrm{mol} \mathrm{h}{ }^{-1}$, respectively). The calculated apparent quantum yields (AQY) for the gyroid $\mathrm{Ta}_{2} \mathrm{O}_{5}$ are 3.7 and $4.6 \%$ for the stabilized and peak rates, respectively among the highest values reported for binary metal oxides optimized only via morphology and structure engineering (see Table S1 and Fig. S7† for calculations). ${ }^{30}$ Most importantly, the peak hydrogen production rate of the mesoporous gyroid $\mathrm{Ta}_{2} \mathrm{O}_{5}$ is about 15 times higher than that of $\mathrm{Ta}_{2} \mathrm{O}_{5}$ SG. This relative increase complies remarkably well with the increase in the BET surface area ( $\sim 15$ times, see Table 1$)$. This linear correlation suggests that activity is not limited by pore diffusion as observed in mesoporous $\mathrm{Ta}_{2} \mathrm{O}_{5}$ photocatalysts with smaller pore sizes. ${ }^{21,22}$ An additional contribution stems from the presence of the gyroid mesoporous network that provides $3 \mathrm{D}$ access to the inner surface (Fig. 1a). The gyroid $\mathrm{Ta}_{2} \mathrm{O}_{5}$ also exhibits excellent chemical and mechanical stability towards harsh UV and ultrasound-assisted treatments (Fig. S5 $\dagger$ ) and
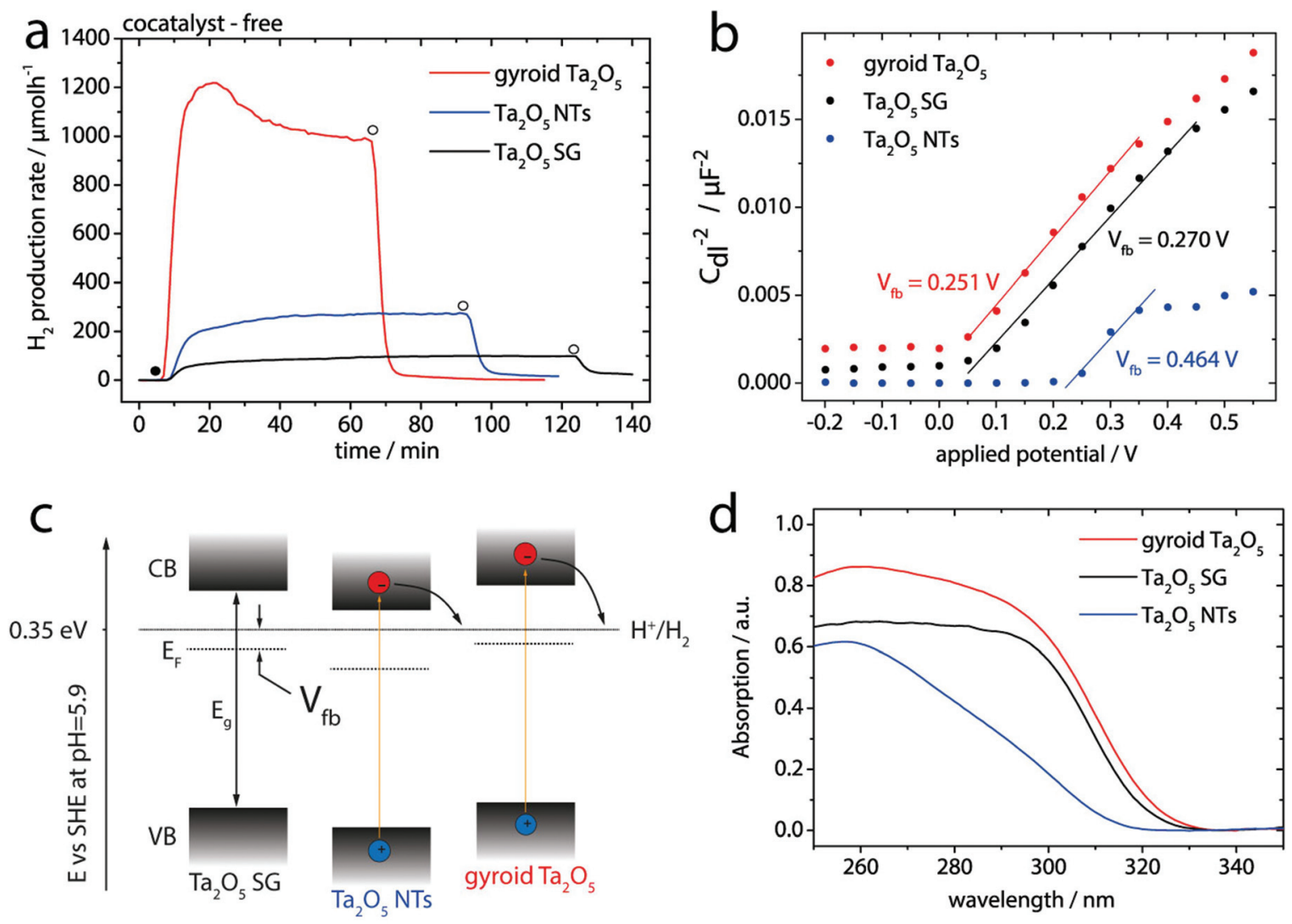

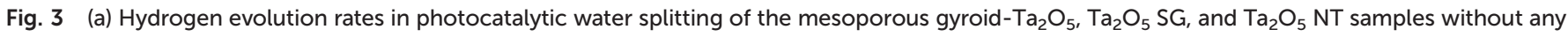

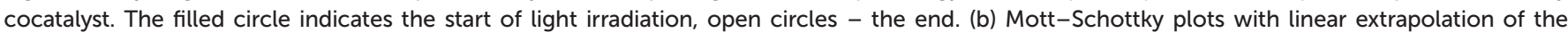

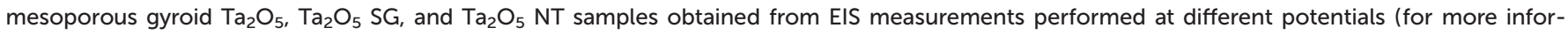

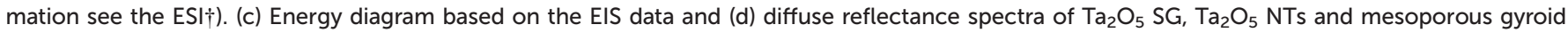
$\mathrm{Ta}_{2} \mathrm{O}_{5}$. 
Table 1 Summary of the material characteristics

\begin{tabular}{|c|c|c|c|c|c|}
\hline & $\begin{array}{l}\text { BET surface } \\
\text { area } / \mathrm{m}^{2} \mathrm{~g}^{-1}\end{array}$ & $\begin{array}{l}\text { Crystallite } \\
\text { size/nm }\end{array}$ & $\begin{array}{l}\mathrm{H}_{2} \text { production } \\
\text { rate/ } \mu \mathrm{mol} \mathrm{h}^{-1}\end{array}$ & $\begin{array}{l}\text { Surface-normalized } \mathrm{H}_{2} \\
\text { production rate } / \mu \mathrm{mol} \mathrm{h}^{-1} \mathrm{~m}^{-2} \mathrm{~g}^{-1}\end{array}$ & $V_{\mathrm{fb}} / V v s . \mathrm{SHE}$ \\
\hline $\mathrm{Ta}_{2} \mathrm{O}_{5} \mathrm{SG}$ & 2.2 & 85.1 & 78 & 35.5 & 0.270 \\
\hline
\end{tabular}

${ }^{a}$ Stabilized rate taken after a few hours.

retains remarkably high activity even at long experimental times, albeit with a gradual slight decrease in the rate, which is not yet fully understood (Fig. S6

For comparison we synthesized mesoporous $\mathrm{Ta}_{2} \mathrm{O}_{5}$ nanotubes $\left(\mathrm{Ta}_{2} \mathrm{O}_{5}\right.$ NTs) with a similar surface area and large accessible inner channels, yet different surface termination by using thick multi-walled carbon nanotubes (CNTs) as a sacrificial hard template as schematically shown in Fig. 4a. In short, CNTs were first coated with $\mathrm{Ta}_{2} \mathrm{O}_{5}$ by means of a hydrothermally-assisted sol-gel process using tantalum(v) ethoxide as a precursor (Fig. S2 $\dagger$ ). ${ }^{31}$ The hybrid was then subjected to heat treatment in ambient air at $700{ }^{\circ} \mathrm{C}$ in order to simultaneously remove CNT templates (their oxidation occurs above $600{ }^{\circ} \mathrm{C}$ ) and crystallize the metal oxide, yielding $\mathrm{Ta}_{2} \mathrm{O}_{5}$ NTs. It is important to note that, although the same calcination procedure was chosen for all samples in this study, crystallization of the $\mathrm{Ta}_{2} \mathrm{O}_{5}$ NTs was strongly affected by the presence of the CNT templates compared to the gyroid $\mathrm{Ta}_{2} \mathrm{O}_{5}$ sample where ISO oxidation occurred at a lower temperature range.

Fig. $4 \mathrm{~b}$ reveals a uniform tubular morphology of $\mathrm{Ta}_{2} \mathrm{O}_{5} \mathrm{NTs}$, well-preserved after the nanocarbon oxidation. The tubes exhibit an inner channel width of about $80 \mathrm{~nm}$ and a wall thickness of about $10 \mathrm{~nm}-$ similar to gyroid $\mathrm{Ta}_{2} \mathrm{O}_{5}$. TEM and electron diffraction (Fig. 4c) confirm complete removal of the nanocarbon (Fig. S3†). From HRTEM it is observed that the tubes consist of large crystalline domains, as indicated by the clear lattice fringes and the single-crystalline nature of the ED (inset). The XRD results in Fig. $2 \mathrm{~b}$ confirm that the nanotubes consist of the crystalline $\beta-\mathrm{Ta}_{2} \mathrm{O}_{5}$ phase, ${ }^{29}$ as for gyroidal $\mathrm{Ta}_{2} \mathrm{O}_{5}$. The Raman spectra in Fig. $2 \mathrm{~d}$ show the typical peaks of $\beta-\mathrm{Ta}_{2} \mathrm{O}_{5}$ without any secondary or impurity phases. The absence of any peak shifts and/or broadening, typically associated with the presence of defects, ${ }^{32}$ corroborates comparable crystal structures in $\mathrm{Ta}_{2} \mathrm{O}_{5}$ NTs and gyroid $\mathrm{Ta}_{2} \mathrm{O}_{5}$ samples.
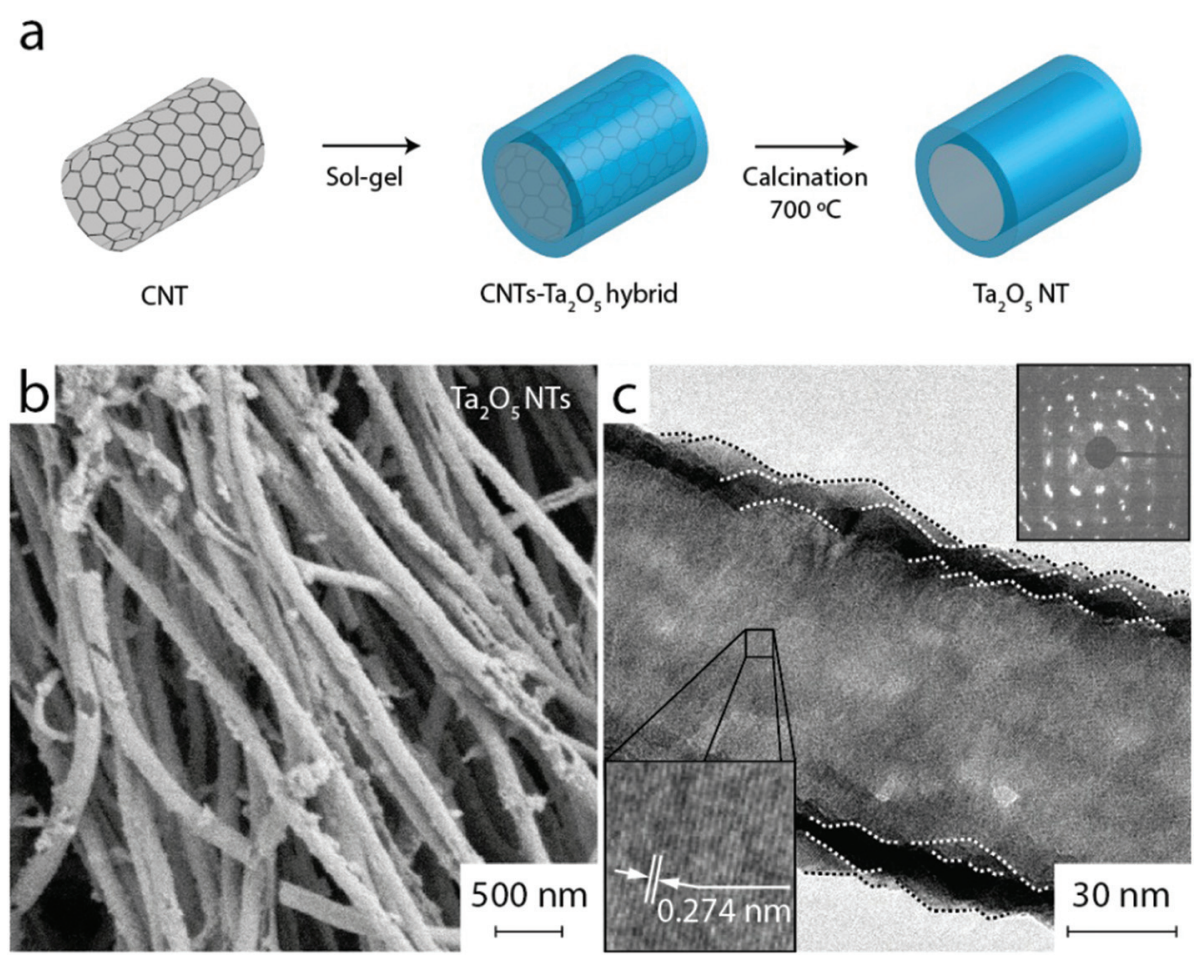

Fig. 4 (a) Schematic of $\mathrm{Ta}_{2} \mathrm{O}_{5}$ NT synthesis using CNTs as sacrificial templates involving sol-gel deposition of oxide onto the CNT surface and subsequent calcination in air at $700{ }^{\circ} \mathrm{C}$ oxidizing away the nanocarbon template and converting the oxide into its crystalline phase. SEM (b) and TEM (c) images of $\mathrm{Ta}_{2} \mathrm{O}_{5}$ NTs. The insets in (c) show the ED pattern of the area in (c) and the magnified region of the sample illustrating fringes of the crystalline structure. 
Finally, the specific surface area of $\mathrm{Ta}_{2} \mathrm{O}_{5}$ NTs is $35 \mathrm{~m}^{2} \mathrm{~g}^{-1}$ according to BET, i.e. also very similar to that of gyroid $\mathrm{Ta}_{2} \mathrm{O}_{5}$. Importantly, the inner surface of the nanotubes is almost entirely accessible for the reactants due to the presence of sufficiently large inner channels.

From these studies we can confirm that $\mathrm{Ta}_{2} \mathrm{O}_{5}$ NTs are similar to gyroid $\mathrm{Ta}_{2} \mathrm{O}_{5}$ in terms of crystal structure, wall thickness/charge transport distance and accessible BET area, yet they differ in terms of their overall morphology and pore structure.

In contrast to the gyroid, the photocatalytic activity for $\mathrm{H}_{2}$ production of $\mathrm{Ta}_{2} \mathrm{O}_{5}$ NTs was only 3-4 times higher $\left(262 \mu \mathrm{mol} \mathrm{h}{ }^{-1}\right)$ than that of $\mathrm{Ta}_{2} \mathrm{O}_{5}$ SG and thus did not correlate with the increase in the surface area. Furthermore, the $\mathrm{Ta}_{2} \mathrm{O}_{5}$ NTs exhibited 4 times lower activity than the gyroid $\mathrm{Ta}_{2} \mathrm{O}_{5}$ (Fig. 3 and Table 1), despite exhibiting a comparable surface area. Consequently, the performance of the NTs with respect to the gyroid is limited by contributions other than pore diffusion.

It is well known that different dominating facets exhibit different surface chemistry/potentials and thus energy barriers (i.e. the flat band potential, $V_{\mathrm{fb}}$ ) at the solid-liquid interface. ${ }^{33}$ We therefore evaluated the flat band potentials of our materials from the Mott-Schottky plots (Fig. 3b) derived from electrochemical impedance spectroscopy (EIS).

The respective values of the flat band potentials, $V_{\mathrm{fb}}$, are summarized in Table 1 . From this analysis the various $\mathrm{Ta}_{2} \mathrm{O}_{5}$ samples exhibit significantly different energy barriers, suggesting a considerable shift in the respective positions of the conduction (CB) and valence (VB) bands. ${ }^{34}$ This is shown schematically in Fig. 3c. For gyroid $\mathrm{Ta}_{2} \mathrm{O}_{5}$ exhibiting the lowest energy barrier, both VB and CB are shifted upwards by $c a$. 0.2 eV compared to $\mathrm{Ta}_{2} \mathrm{O}_{5}$ NTs, resulting in a stronger driving force for $\mathrm{H}^{+}$reduction and, concomitantly, a higher photocatalytic rate.

The difference in the flat band potential, i.e. surface chemistry, can be explained by a closer look at XRD (Fig. 2b). The data reveal that the relative intensities of the (100), (011) and (111) peaks are different between the two samples. This suggests the different surface termination as a result of different predominant growth directions within the crystalline walls, with a higher proportion towards (111) in gyroid $\mathrm{Ta}_{2} \mathrm{O}_{5}$ than in the NTs. HRTEM further confirms a different surface termination, i.e. a rather smooth surface for gyroid $\mathrm{Ta}_{2} \mathrm{O}_{5}$ compared with many sharp edges for $\mathrm{Ta}_{2} \mathrm{O}_{5}$ NTs (Fig. 1e and $4 \mathrm{c}$, dashed lines). We believe that this difference originates from the strong influence of the CNT template on the crystallization during the heat treatment of $\mathrm{Ta}_{2} \mathrm{O}_{5}$ NTs by e.g. affecting crystal nucleation and growth. ${ }^{31}$

We speculate that the observed differences in surface termination are directly linked to the $V_{\mathrm{fb}}$ values. In this picture, the superior activity for the mesoporous gyroid $\mathrm{Ta}_{2} \mathrm{O}_{5}$ would, at least in part, be due to a higher fraction of more active surface facets at the solid-liquid interface.

Another effect that may influence the performance could stem from the ordered mesoporous gyroidal network. Diffuse reflectance spectroscopy (DRS; Fig. 3d) revealed that all three
$\mathrm{Ta}_{2} \mathrm{O}_{5}$ samples share a similar absorption edge at about $320 \mathrm{~nm}$, which corresponds to a previously reported band gap value of about $3.9 \mathrm{eV} \cdot{ }^{35}$ However, the gyroid $\mathrm{Ta}_{2} \mathrm{O}_{5}$ exhibits a considerably stronger absorption around the UVB range (250-310 nm), which accounts for high energy photons of 4 to $5 \mathrm{eV}$. Superior light trapping in the UVB region and thus photoexcitation to higher-energy levels can additionally enhance catalyst's activity towards $\mathrm{H}^{+}$reduction. ${ }^{36}$ Although the origin of this surprising optical effect is not yet well understood and clearly warrants more detailed investigations, it is likely connected to the presence of well-ordered gyroidal mesopores.

\section{Conclusion}

We report on the synthesis of well-ordered mesoporous tantalum oxides with an alternating gyroidal pore structure exhibiting large pore diameters $(>20 \mathrm{~nm})$ with a $3 \mathrm{D}$ accessible pore matrix and extended single-crystalline domains as well as of unique $\mathrm{Ta}_{2} \mathrm{O}_{5}$ nanotubes using tailor-made block-copolymers and carbon nanotubes as templates, respectively. In particular, we have developed a synthetic protocol using a tailor-made triblock-terpolymer, i.e. poly(isoprene)- $b$-poly(styrene)- $b$-poly (ethylene oxide), as a structure-directing agent that allows for tuning of the pore size and architecture.

The resulting $\mathrm{Ta}_{2} \mathrm{O}_{5}$ gyroids have yielded high photocatalytic activities of up to $20 \mathrm{mmol} \mathrm{h} \mathrm{h}^{-1} \mathrm{~g}^{-1}$ towards hydrogen evolution with the apparent quantum yield of $4.6 \%$ without the use of a cocatalyst. In particular, we observed that the peak rate of the gyroid yielded about the same value as the sol-gel sample when corrected to the specific surface area, while the stabilized rate still retained about $80 \%$ of its peak value after several hours. This confirms that the pore diffusion has been successfully eliminated.

We further demonstrate that the nature of the template affects the crystallization and surface chemistry of $\mathrm{Ta}_{2} \mathrm{O}_{5}$ (e.g. injection barriers) and consequently the photocatalytic performance of the resulting mesostructures.

This work utilizes a fundamental materials design strategy to evaluate and eliminate diffusion limitations that are inherent to mesoporous materials and play a crucial role in liquid phase photocatalysis and photoelectrochemistry with both UV- and visible-light active materials. It further provides invaluable mechanistic insight into key criteria for photocatalyst design that is applicable also to visible-light active photocatalysis. We believe that this work will help in establishing pore and surface engineering as a powerful tool for designing high-performance catalysts and electrodes for various applications related to energy, environment and health.

\section{Acknowledgements}

This work was supported by the National Science Foundation (NSF) Single Investigator Award (DMR-1104773). This work 
made use of the Cornell Center for Materials Research Shared Facilities, which are supported through the NSF MRSEC program (DMR-1120296). This work is based upon research conducted at the Cornell High Energy Synchrotron Source (CHESS), which is supported by the National Science Foundation and the National Institutes of Health/National Institute of General Medical Sciences under the NSF award DMR-0936384. The research leading to these results has received funding from the European Union Seventh Framework Programme under the grant agreement No. 310184, CARINHYPH project. AC is grateful for the funding provided by the graduate school of chemistry at the University of Münster (GSC-MS). We further thank Huating $\mathrm{Hu}$ for Raman measurements.

\section{References}

1 T. Hisatomi, J. Kubota and K. Domen, Chem. Soc. Rev., 2014, 43, 7520-7535.

2 L. Barreto, A. Makihira and K. Riahi, Int. J. Hydrogen Energy, 2003, 28, 267-284.

3 S. S. Penner, Energy, 2006, 31, 33-43.

4 W. McDowall and M. Eames, Energy Policy, 2006, 34, 12361250.

5 M. Ball and M. Wietschel, The Hydrogen Economy: Opportunities and Challenges, Cambridge University Press, 2009.

6 M. Nedelcu, S. Guldin, M. C. Orilall, J. Lee, S. Hüttner, E. J. W. Crossland, S. C. Warren, C. Ducati, P. R. Laity, D. Eder, U. Wiesner, U. Steiner and H. J. Snaith, J. Mater. Chem., 2010, 20, 1261-1268.

7 J. Zhu and M. Zäch, Curr. Opin. Colloid Interface Sci., 2009, 14, 260-269.

8 A. Corma, Chem. Rev., 1997, 97, 2373-2420.

9 A. Taguchi and F. Schüth, Microporous Mesoporous Mater., 2005, 77, 1-45.

10 A. A. Ismail and D. W. Bahnemann, J. Mater. Chem., 2011, 21, 11686-11707.

11 M. C. Orilall and U. Wiesner, Chem. Soc. Rev., 2011, 40, 520-535.

12 D. M. Antonelli and J. Y. Ying, Chem. Mater., 1996, 8, 874881.

13 W. Lee, R. Scholz, K. Nielsch and U. Gösele, Angew. Chem., Int. Ed., 2005, 44, 6050-6054.

14 L. Li, M. Krissanasaeranee, S. W. Pattinson, M. Stefik, U. Wiesner, U. Steiner and D. Eder, Chem. Commun., 2010, 46, 7620-7622.
15 P. Docampo, M. Stefik, S. Guldin, R. Gunning, N. A. Yufa, N. Cai, P. Wang, U. Steiner, U. Wiesner and H. J. Snaith, Adv. Energy Mater., 2012, 2, 676-682.

16 H. Kato, K. Asakura and A. Kudo, J. Am. Chem. Soc., 2003, 125, 3082-3089.

17 M. Hara, G. Hitoki, T. Takata, J. N. Kondo, H. Kobayashi and K. Domen, Catal. Today, 2003, 78, 555-560.

18 Y. Takahara, J. N. Kondo, T. Takata, D. Lu and K. Domen, Chem. Mater., 2001, 13, 1194-1199.

19 J. N. Kondo, Y. Takahara, B. Lee, D. Lu and K. Domen, Top. Catal., 2002, 19, 171-177.

20 G. Guo and J. Huang, Mater. Lett., 2011, 65, 64-66.

21 Y. Noda, B. Lee, K. Domen and J. N. Kondo, Chem. Mater., 2008, 20, 5361-5367.

22 L. Guo, H. Hagiwara, S. Ida, T. Daio and T. Ishihara, ACS Appl. Mater. Interfaces, 2013, 5, 11080-11086.

23 M. Stefik, S. Wang, R. Hovden, H. Sai, M. W. Tate, D. A. Muller, U. Steiner, S. M. Gruner and U. Wiesner, J. Mater. Chem., 2012, 22, 1078-1087.

24 D. Eder and A. H. Windle, Adv. Mater., 2008, 20, 1787-1793. 25 M. Stefik, J. Song, H. Sai, S. Guldin, P. Boldrighini, M. C. Orilall, U. Steiner, S. M. Gruner and U. Wiesner, J. Mater. Chem. A, 2015, 3, 11478-11492.

26 J. Chatterjee, S. Jain and F. S. Bates, Macromolecules, 2007, 40, 2882-2896.

27 C. A. Tyler, J. Qin, F. S. Bates and D. C. Morse, Macromolecules, 2007, 40, 4654-4668.

28 S. W. Robbins, H. Sai, F. J. DiSalvo, S. M. Gruner and U. Wiesner, ACS Nano, 2014, 8, 8217-8223.

29 L. A. Aleshina and S. V. Loginova, Crystallogr. Rep., 2002, 47, 415-419.

30 X. Li, J. Yu, J. Low, Y. Fang, J. Xiao and X. Chen, J. Mater. Chem. A, 2015, 3, 2485-2534.

31 A. S. Cherevan, P. Gebhardt, C. J. Shearer, M. Matsukawa, K. Domen and D. Eder, Energy Environ. Sci., 2014, 7, 791796.

32 D. Eder, M. S. Motta and A. H. Windle, Acta Mater., 2010, 58, 4406-4413.

33 M. Grätzel, Nature, 2001, 414, 338-344.

34 L. Liao, Q. Zhang, Z. Su, Z. Zhao, Y. Wang, Y. Li, X. Lu, D. Wei, G. Feng, Q. Yu, X. Cai, J. Zhao, Z. Ren, H. Fang, F. Robles-Hernandez, S. Baldelli and J. Bao, Nat. Nanotechnol., 2014, 9, 69-73.

35 W.-J. Chun, A. Ishikawa, H. Fujisawa, T. Takata, J. N. Kondo, M. Hara, M. Kawai, Y. Matsumoto and K. Domen, J. Phys. Chem. B, 2003, 107, 1798-1803.

36 H. Kato and A. Kudo, J. Phys. Chem. B, 2001, 105, 42854292. 